\title{
Block-oriented identification of Hammerstein/Wiener-models using the RLS-algorithm
}

\author{
Martin KozeK, Christoph HametneR \\ Vienna University of Technology, Gußhausstraße 27-29, 1040 Vienna, Austria; kozek@impa.tuwien.ac.at
}

\begin{abstract}
A new method for identifying Hammerstein/Wiener models is proposed, where the parameters of a piecewise linear characteristic and a linear transfer function are estimated by recursive least-squares (RLS). Using smooth validity functions and an adaptive algorithm for optimal partitioning of the local linear models an efficient and accurate model can be identified.
\end{abstract}

\section{Introduction}

Hammerstein and Wiener models are composed of a non-linear static characteristic followed by a linear dynamic transfer function, and vice versa [1]. The main problem with identifying the parameters of these systems lies within the multiplicative coupling of parameters. Consequently, several methods with different choice of parameterization of the non-linear characteristic have been proposed, all of them combining linear and non-linear optimization techniques $[1,2]$.

The algorithm proposed here is related to a local linear model approach [1]. After selecting an appropriate partition of input or output data, respectively, a difference equation with bias term is estimated using the RLS-algorithm. The parameters of the linear transfer function are valid for all segments, while each gain and bias parameter is only valid inside one segment. Therefore, the non-linear characteristic is given by a piecewise linear function.

\section{Wiener model}

Inside each data segment the following discrete-time linear model structure is assumed to be valid:

$$
y(k)+a_{1} y(k-1)+\ldots+a_{n} y(k-n)=b_{1} u(k-1)+\ldots+b_{m} u(k-m)+c .
$$

Here $y(k)$ is the model output, $u(k)$ is the model input, the unknown parameters are constituted by the $a_{i}$ (coefficients of the denominator polynomial), the $b_{i}$ (coefficients of the numerator polynomial), and the bias term $c . n$ and $m$ are the respective polynomial orders. The gain $K$ and the intercept $d$ of the $i$-th local linear model are given by $y(k)=K_{i} v(k)+d_{i}$, where $v(k)$ is the intermediate variable of the Wiener model (see fig.1,a)+b)).

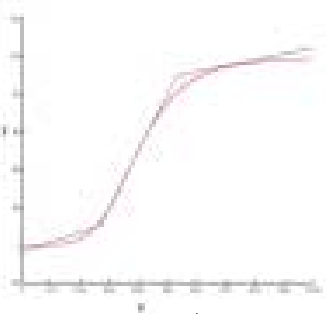

a)

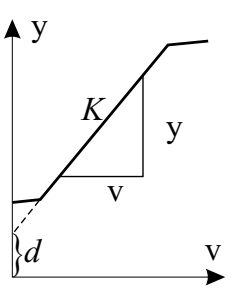

b)

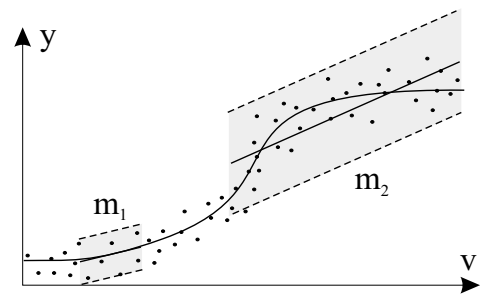

c)

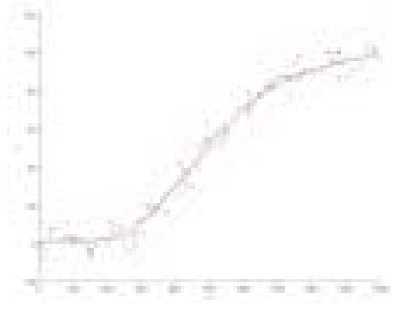

d)

Figure 1: Piecewise linear characteristic. a) Approximation of continuous function. b) Parameters of local model. c) Criterion for optimal partitioning. d) Identified characteristic from noisy data.

The resulting parameter vector for the RLS-algorithm is then defined as

$$
\underline{\theta}=\left[\begin{array}{lllll}
a_{1} & a_{2} & K_{i} b_{1} & K_{i} b_{2} & d_{i}\left(1+a_{1}+a_{2}\right)
\end{array}\right]^{T},
$$


which allows a unique computation of the original parameters in the case of a transfer function with unity gain. It is important to notice that only monotonically increasing or decreasing characteristics can be identified due to the unique output mapping. Nevertheless, hard non-linearities such as discontinuities are also covered.

\section{Hammerstein model}

The Hammerstein model is given by

$$
y(k)+a_{1} y(k-1)+\ldots+a_{n} y(k-n)=b_{1} w(k-1)+\ldots+b_{m} w(k-m)+c
$$

where $w(k)=\kappa u(k)+\delta$ is the intermediate variable. In this case the non-linear characteristic is not restricted to monotonous functions. All other computations are completely analogous to the Wiener model.

\section{Validity Functions}

All linear models are valid only inside their specific data segment. This corresponds to a boxcar function for each linear model. In order to smoothen the model transitions, alternate validity functions $g(v)$ can be utilized, such as $g(v)=\exp \left[\left(\frac{2(v-\beta}{\alpha}\right)^{\gamma} \ln (0.5)\right]$, where the $\alpha, \beta$, and $\gamma$ are shape parameters.

\section{Adaptive optimal partitioning}

In order to relieve the user from choosing a good partition, an approximate statistic for the output variance may be computed from the estimated parameter covariance matrix [3]. By recursively computing the prediction interval of the output for each additional data, the proportion between the magnitude of the prediction interval and the length of the input segment is defined as criterion for the optimal partitioning (similar to the correlation coefficient). In fig.1,c) a small local model $\left(m_{1}\right)$ and a large local model $\left(m_{2}\right)$ with poor performance are depicted. The optimal model size is detected by iteratively computing the criterion and searching for a minimum.

\section{Results for a 2nd-order system}

A 2nd-order Wiener model with a sigmoid characteristic was identified from 100 samples. The true nonlinearity (black), the noisy samples (black x), the estimated model (red, solid), and the prediction intervals (red, dashed) can be seen in fig.1,d). Although only 3 local linear models have been used, a good overall fit and narrow prediction intervals result. The partition was automatically optimized by the algorithm.

\section{Conclusion}

A new method for identifying block-oriented models has been proposed, utilizing the RLS-algorithm for estimation of the parameters of a piecewise linear characteristic and a linear transfer function. Smooth validity functions guarantee a continuous characteristic, and a partitioning algorithm ensures optimal fit. Simulations prove the efficiency and performance of the proposed method.

\section{References}

[1] O. Nelles. Nonlinear System Identification, Springer, 2001.

[2] M. Kozek and N. Jovanovic. Identification of Wiener/Hammerstein Nonlinear Systems with Extended Kalman Filters, Proc. IEEE ACC02, May 8-10, 2002, Anchorage, Alaska.

[3] L. Ljung and T. Söderström. Theory and Practice of Recursive Identification, MIT Press, Cambridge, 1983. 